\title{
@creative
}

ISSN 1855-3966 (printed edn.), ISSN 1855-3974 (electronic edn.)

\author{
ARS MATHEMATICA CONTEMPORANEA 15 (2018) 53-65 \\ https://doi.org/10.26493/1855-3974.1161.3b9 \\ (Also available at http://amc-journal.eu)
}

\section{On prime-valent symmetric graphs of square-free order*}

\author{
Jiangmin Pan ${ }^{\dagger}$ \\ School of Statistics and Mathematics, Yunnan University of Finance and Economics, \\ Kunming, Yunnan, P. R. China \\ Bo Ling \\ School of Mathematics and Computer Science, Yunnan Minzu University, \\ Kunming, Yunnan, P. R. China \\ Suyun Ding \\ School of Mathematics and Statistics, Yunnan University, \\ Kunming, Yunnan, P. R. China
}

Received 16 July 2016, accepted 27 May 2017, published online 10 November 2017

\begin{abstract}
Symmetric graphs of valencies 3, 4 and 5 and square-free order have been classified in the literature. In this paper, we will present a complete classification of symmetric graphs of square-free order and any prime valency which admit a soluble arc-transitive group, and a complete classification of 7 -valent symmetric graphs of square-free order.
\end{abstract}

Keywords: Symmetric graph, normal quotient graph, automorphism group.

Math. Subj. Class.: 20B15, 20B30, 05C25

\section{Introduction}

Throughout the paper, graphs considered are assumed to be undirected and simple with valency at least three.

For a graph $\Gamma$, denote by $V \Gamma$ and $A \Gamma$ the vertex set and arc set of $\Gamma$ respectively, denote by Aut $\Gamma$ the full automorphism group of $\Gamma$, and denote by $\Gamma(\alpha)$ the set of neighbors of a

\footnotetext{
* The authors are very grateful to the referees for their valuable comments. This work was partially supported by the National Natural Science Foundation of China (11461007, 11231008).

${ }^{\dagger}$ Corresponding author.

E-mail addresses: jmpan@ynu.edu.cn (Jiangmin Pan), bolinggxu@163.com (Bo Ling), 1328897542@qq.com (Suyun Ding)
}

(a)(i) This work is licensed under http://creativecommons.org/licenses/by/3.0/ 
vertex $\alpha$ in $\Gamma$. Then $\Gamma$ is called $X$-vertex-transitive or $X$-arc-transitive, with $X \leq$ Aut $\Gamma$, if $X$ is transitive on $V \Gamma$ or $A \Gamma$ respectively. An arc-transitive graph is also called a symmetric graph. In particular, $\Gamma$ is called arc-regular if Aut $\Gamma$ is regular on $A \Gamma$.

For a positive integer $s$, an $s$-arc of a graph $\Gamma$ is a sequence $v_{0}, v_{1}, \ldots, v_{s}$ of $s+1$ vertices such that $v_{i-1}, v_{i}$ are adjacent for $1 \leq i \leq s$ and $v_{i-1} \neq v_{i+1}$ for $1 \leq i \leq s-1$. If $\Gamma$ has an $s$-arc and $X \leq$ Aut $\Gamma$ is transitive on the set of $s$-arcs of $\Gamma$, then $\Gamma$ is called $(X, s)$-arc-transitive. If $\Gamma$ is (Aut $\Gamma, s)$-arc-transitive but not (Aut $\Gamma, s+1)$-arc-transitive, then $\Gamma$ is simply called $s$-transitive.

Characterizing symmetric graphs was initiated by a nice result of Tutte (1949) which says that there exists no $s$-arc-transitive cubic graph with $s \geq 6$. This result was generalized by Weiss [27] who proved that there is no $s$-arc-transitive graph with $s \geq 8$ of valency at least 3 . Since then, studying transitive graphs has been one of the main topics in algebraic graph theory, and numerous results have been obtained. In particular, transitive graphs of square-free order (not divisible by the square of a prime) have received considerable attention; for example, symmetric graphs of valencies 3, 4 and 5 and square-free order have been classified by [16, 17] and [6] respectively, and arc-regular graphs of square-free order and prime valency have been determined by [9]. The main purpose of this paper is to give a complete classification of symmetric graphs of square-free order and prime valency admitting a soluble arc-transitive group, and a complete classification of 7-valent symmetric graphs of square-free order.

The terminology and notation used in this paper are standard. For example, we denote by $J_{1}$ the Janko simple group, by HS the Higman-Sims simple group, and by $\mathrm{M}_{n}$, with $n=11,12,22,23,24$, the five Mathieu simple groups. For a positive integer $m$, denote by $A_{m}$ and $S_{m}$ the alternating group and symmetric group of degree $m$, and by $\mathbb{Z}_{m}, \mathrm{~F}_{m}$ and $\mathrm{D}_{m}$ (with $m$ even) the cyclic group, Frobenius group and dihedral group of order $m$ respectively. Given two groups $N$ and $H$, denote by $N \times H$ the direct product of $N$ and $H$, by $N . H$ an extension of $N$ by $H$, and if such an extension is split, then we write $N: H$ instead of $N . H$.

A graph $\Gamma$ is called a Cayley graph if there exists a group $G$ and a subset $S \subseteq G \backslash\{1\}$ with $S=S^{-1}:=\left\{s^{-1} \mid s \in S\right\}$ such that the vertex set $V \Gamma=G$ and a vertex $x$ is adjacent to a vertex $y$ if and only if $y x^{-1} \in S$. This Cayley graph is denoted by Cay $(G, S)$. The following Cayley graphs of dihedral groups give rise to an infinite family of prime-valent symmetric graphs, where the first two letters 'CD' of the name of the graph $\mathrm{CD}_{2 m, p, k}$ stand for 'Cayley graph of a dihedral group'.

Example 1.1. Let $G=\left\langle a, b \mid a^{m}=b^{2}=1, a^{b}=a^{-1}\right\rangle \cong \mathrm{D}_{2 m}$ with $m$ a positive integer, and let $p$ be an odd prime and $k$ a solution of the equation

$$
x^{p-1}+x^{p-2}+\cdots+x+1 \equiv 0 \quad(\bmod m) .
$$

Set

$$
\mathrm{CD}_{2 m, p, k}=\operatorname{Cay}\left(G,\left\{b, a b, a^{k+1} b, \ldots, a^{k^{p-2}+k^{p-3}+\cdots+k+1} b\right\}\right) .
$$

The following theorem determines the prime-valent symmetric graphs of square-free order which admit a soluble arc-transitive automorphism group. We remark that cubic graphs which admit a soluble edge-transitive or arc-transitive automorphism group have been characterized by [20] and [8], respectively. 
Theorem 1.2. Let $\Gamma$ be a connected $p$-valent symmetric graph of square-free order $n$ with $p$ an odd prime, and suppose that $\Gamma$ admits a soluble arc-transitive automorphism group G. Then either

(1) $\Gamma \cong \mathrm{K}_{p, p}$, and $G \cong\left(\left(\left(\mathbb{Z}_{p}: \mathbb{Z}_{l_{1}}\right) \times\left(\mathbb{Z}_{p}: \mathbb{Z}_{l_{2}}\right)\right) \cdot \mathbb{Z}_{r}\right) \cdot \mathbb{Z}_{2} \leq \mathrm{S}_{p} 2 \mathbb{Z}_{2}$, where $l_{i} r \mid p-1$ for $i=1,2$; or

(2) $\Gamma \cong \mathrm{CD}_{n, p, k}, n=2 \cdot p^{s} p_{1} p_{2} \cdots p_{t}$ and $G=$ Aut $\Gamma \cong \mathrm{D}_{n}: \mathbb{Z}_{p}$, where $0 \leq s \leq 1$, $t \geq 1$, and $p_{1}, p_{2}, \ldots, p_{t}$ are distinct primes such that $p \mid p_{i}-1$ for $i=1,2, \ldots, t$. Further, there are exactly $(p-1)^{t-1}$ non-isomorphic such graphs of order $n$.

The next theorem present a complete classification of 7 -valent symmetric graphs of square-free order, where the graph $\mathcal{C}_{330}$ in Table 1 is introduced in Example 3.2 for convenience.

Theorem 1.3. Let $\Gamma$ be a connected 7-valent symmetric graph of square-free order $n$. Then one of the following statements holds.

(1) $\Gamma \cong \mathrm{CD}_{n, 7, k}$, and the tuple $(n$, Aut $\Gamma)$ is as in part (2) of Theorem 1.2 with $p=7$.

(2) The triple $(\Gamma, n$, Aut $\Gamma)$ lies in Table 1.

(3) Aut $\Gamma \cong \operatorname{PSL}(2, p)$ or $\operatorname{PGL}(2, p)$, where $p \geq 13$ is a prime such that $p\left(p^{2}-1\right)$ $2^{25} \cdot 3^{4} \cdot 5^{2} \cdot 7 n$.

Table 1: Two 'sporadic' 7-valent symmetric graphs

\begin{tabular}{crrcccl} 
Row & $\Gamma$ & $n$ & Aut $\Gamma$ & $(\text { Aut } \Gamma)_{\alpha}$ & Transitivity & Remark \\
\hline \hline 1 & $\mathrm{~K}_{7,7}$ & 14 & $\mathrm{~S}_{7} \backslash \mathbb{Z}_{2}$ & $\mathrm{~S}_{7} \cdot \mathrm{S}_{6}$ & 3-transitive & bipartite \\
\hline 2 & $\mathcal{C}_{330}$ & 330 & $\mathrm{M}_{22} \cdot \mathbb{Z}_{2}$ & $\mathbb{Z}_{2}^{4}: \mathrm{SL}(3,2)$ & 2-transitive & not bipartite
\end{tabular}

Remark 1.4. Graphs appearing in part (3) of Theorem 1.3 can be expressed as coset graphs of $\operatorname{PSL}(2, p)$ or $\operatorname{PGL}(2, p)$ (refer to [10] for the definition of the coset graph). However, it seems infeasible to determine all the possible values of $p$ (and so the corresponding symmetric graphs $\Gamma$ ) for general square-free integer $n$.

\section{Preliminaries}

In this section, we introduce some preliminary results that will be used later.

For a group $G$ with a subgroup $H$, let $C_{G}(H)$ and $N_{G}(H)$ denote the centralizer and normalizer of $H$ in $G$, respectively.

Lemma 2.1 ([14, Ch. I, Lemma 4.5]). Let $G$ be a group and $H$ a subgroup of $G$. Then $N_{G}(H) / C_{G}(H) \lesssim \operatorname{Aut}(H)$.

For a group $G$, the largest nilpotent normal subgroup of $G$ is called the Fitting subgroup of $G$. Clearly, the Fitting subgroup is a characteristic subgroup. The next lemma gives a property of the Fitting subgroup of soluble groups.

Lemma 2.2 ([26, P. 30, Corollary]). Let $F$ be the Fitting subgroup of a soluble group $G$. Then $F \neq 1$ and $C_{G}(F) \leq F$. 
The maximal subgroups of the simple group $\operatorname{PSL}(2, q)$ are known, see [5, Section 239].

Lemma 2.3. Let $T=\operatorname{PSL}(2, q)$, where $q=p^{n} \geq 5$ with $p$ a prime. Then a maximal subgroup of $T$ is isomorphic to one of the following groups, where $d=(2, p-1)$.

(1) $\mathrm{D}_{\frac{2(q-1)}{d}}$, where $q \neq 5,7,9,11$;

(2) $\mathrm{D}_{\frac{2(q+1)}{d}}$, where $q \neq 7,9$;

(3) $\mathbb{Z}_{p}^{n}: \mathbb{Z}_{\frac{q-1}{d}}$;

(4) $\mathrm{A}_{4}$, where $q=5$, or $q=p \equiv 3,13,27,37(\bmod 40)$;

(5) $\mathrm{S}_{4}$, where $q=p \equiv \pm 1(\bmod 8)$;

(6) $\mathrm{A}_{5}$, where $q=p \equiv \pm 1(\bmod 5)$, or $q=p^{2} \equiv-1(\bmod 5)$ with $p$ an odd prime;

(7) $\operatorname{PSL}(2, r)$, where $q=r^{m}$ with $m$ an odd prime;

(8) $\operatorname{PGL}(2, r)$, where $q=r^{2}$.

By [2, Theorem 2], one may easily derive the maximal subgroups of $\operatorname{PGL}(2, p)$.

Lemma 2.4. Let $T=\operatorname{PGL}(2, p)$ with $p \geq 5$ a prime. Then a maximal subgroup of $T$ is isomorphic to one of the following groups:

(1) $\mathbb{Z}_{p}: \mathbb{Z}_{p-1}$;

(2) $\mathrm{D}_{2(p+1)}$;

(3) $\mathrm{D}_{2(p-1)}$, where $p \geq 7$;

(4) $\mathrm{S}_{4}$, where $p \equiv \pm 3(\bmod 8)$;

(5) $\operatorname{PSL}(2, p)$.

A group $G$ is called perfect if $G=G^{\prime}$, the commutator subgroup; and an extension $G=N . H$ is called a central extension if $N \subseteq Z(G)$, the center of $G$. If a group $G$ is perfect and $G / Z(G)$ is isomorphic to a simple group $T$, then $G$ is called a covering group of $T$. Schur [25] showed that a simple (and, more generally, perfect) group $T$ possesses a universal covering group $G$ with the property that every covering group of $T$ is a homomorphic image of $G$, in this case, the center $Z(G)$ is called the Schur multiplier of $T$, denoted by $\operatorname{Mult}(T)$, see [12, P. 43]. The Schur multipliers of nonabelian simple groups are known (see [12, P. 302]), and the following lemma is easy to prove (see [23, Lemma 2.11]).

Lemma 2.5. Let $G=N . T$, where $N$ is a cyclic group and $T$ is a nonabelian simple group. Then $G=N . T$ is a central extension. Further, $G=N G^{\prime}$ and $G^{\prime}=M . T$, where $M$ is contained in $G^{\prime} \cap N$ and is isomorphic to a subgroup of $\operatorname{Mult}(T)$.

The following lemma characterizes the vertex stabilizers of 7-valent symmetric graphs, see [13, Theorem 1.1].

Lemma 2.6. Let $\Gamma$ be a connected 7 -valent $(X, s)$-arc-transitive graph, where $X \leq$ Aut $\Gamma$ and $s \geq 1$. Then one of the following holds, where $\alpha \in V \Gamma$.

(1) If $X_{\alpha}$ is soluble, then $s \leq 3$ and $\left|X_{\alpha}\right| \mid 252$. Further, the couple $\left(s, X_{\alpha}\right)$ is listed in Table 2. 
Table 2: Soluble vertex-stabilizers of 7-valent-symmetric graphs.

\begin{tabular}{c||c|c|c}
$s$ & 1 & 2 & 3 \\
\hline$X_{\alpha}$ & $\mathbb{Z}_{7}, \mathrm{D}_{14}, \mathrm{~F}_{21}, \mathrm{D}_{14} \times \mathbb{Z}_{2}$, & $\operatorname{AGL}(1,7), \operatorname{AGL}(1,7) \times \mathbb{Z}_{2}$, & $\operatorname{AGL}(1,7) \times \mathbb{Z}_{6}$ \\
& $\mathrm{~F}_{21} \times \mathbb{Z}_{3}$ & $\operatorname{AGL}(1,7) \times \mathbb{Z}_{3}$ &
\end{tabular}

Table 3: Insoluble vertex-stabilizers of 7-valent symmetric graphs.

\begin{tabular}{c||c|c}
$s$ & 2 & 3 \\
\hline$X_{\alpha}$ & $\operatorname{PSL}(3,2), \operatorname{ASL}(3,2)$, & $\operatorname{PSL}(3,2) \times \mathrm{S}_{4}, \mathrm{~A}_{7} \times \mathrm{A}_{6}$, \\
& $\operatorname{ASL}(3,2) \times \mathbb{Z}_{2}, \mathrm{~A}_{7}, \mathrm{~S}_{7}$ & $\mathrm{~S}_{7} \times \mathrm{S}_{6},\left(\mathrm{~A}_{7} \times \mathrm{A}_{6}\right): \mathbb{Z}_{2}$, \\
& & $\mathbb{Z}_{2}^{6}:(\mathrm{SL}(2,2) \times \mathrm{SL}(3,2))$, \\
& & $\mathbb{Z}_{2}^{20}:(\mathrm{SL}(2,2) \times \mathrm{SL}(3,2))$ \\
\hline$\left|X_{\alpha}\right|$ & $2^{3} \cdot 3 \cdot 7,2^{6} \cdot 3 \cdot 7$, & $2^{6} \cdot 3^{2} \cdot 7,2^{6} \cdot 3^{4} \cdot 5^{2} \cdot 7$, \\
& $2^{7} \cdot 3 \cdot 7,2^{3} \cdot 3^{2} \cdot 5 \cdot 7,2^{4} \cdot 3^{2} \cdot 5 \cdot 7$ & $2^{8} \cdot 3^{4} \cdot 5^{2} \cdot 7,2^{7} \cdot 3^{4} \cdot 5^{2} \cdot 7$, \\
& & $2^{10} \cdot 3^{2} \cdot 7,2^{24} \cdot 3^{2} \cdot 7$
\end{tabular}

(2) If $X_{\alpha}$ is insoluble, then $\left|X_{\alpha}\right| \mid 2^{24} \cdot 3^{4} \cdot 5^{2} \cdot 7$. Further, the couple $\left(s, X_{\alpha}\right)$ lies in Table 3.

Analyzing a graph in terms of its normal quotients is a typical method for studying vertex-transitive graphs. Let $\Gamma$ be an $X$-vertex-transitive graph with $X \leq$ Aut $\Gamma$, and suppose that $X$ has a normal subgroup $N$ which is intransitive on $V \Gamma$. Denote by $V \Gamma_{N}$ the set of all $N$-orbits on $V \Gamma$. Then the normal quotient graph $\Gamma_{N}$ of $\Gamma$ induced by $N$ is defined as the graph with vertex set $V \Gamma_{N}$, and $B$ is adjacent to $C$ in $\Gamma_{N}$ if and only if there exist vertices $\beta \in B$ and $\gamma \in C$ such that $\beta$ is adjacent to $\gamma$ in $\Gamma$. In particular, if for any adjacent vertices $B$ and $C$ in $V \Gamma_{N}$, the induced subgraph $[B, C] \cong m \mathrm{~K}_{2}$ is a perfect matching, where $m=|B|=|C|$, then $\Gamma$ is called a regular cover (or normal cover) of $\Gamma_{N}$.

The following theorem gives a basic reduction method for studying vertex-transitive locally primitive graphs (see [18, Lemma 2.5]), which slightly improves a nice result of Praeger [24, Theorem 4.1]. Recall that, a graph $\Gamma$ is called $X$-locally primitive if the vertex stabilizer $X_{\alpha}$ acts primitively on the neighbour set $\Gamma(\alpha)$ for each $\alpha \in V \Gamma$. Obviously, symmetric graphs with odd prime valency are locally primitive.

Theorem 2.7. Let $\Gamma$ be an $X$-vertex-transitive locally primitive graph, where $X \leq$ Aut $\Gamma$, and let $N \triangleleft X$ have at least three orbits on $V \Gamma$. Then the following statements hold.

(i) $N$ is semi-regular on $V \Gamma, X / N \leq \operatorname{Aut} \Gamma_{N}$, and $\Gamma$ is a regular $N$-cover of $\Gamma_{N}$;

(ii) $X_{\alpha} \cong(X / N)_{\gamma}$, where $\alpha \in V \Gamma$ and $\gamma \in V \Gamma_{N}$;

(iii) $\Gamma$ is $(X, s)$-arc-transitive if and only if $\Gamma_{N}$ is $(X / N, s)$-arc-transitive, where $1 \leq s \leq 5$ or $s=7$.

Symmetric graphs of prime-valency and order twice a prime are known, see [3].

Lemma 2.8. Let $\Gamma$ be a connected symmetric graph of odd prime valency $p$ and order $2 r$ with $r$ a prime. Then one of the following statements holds. 
(1) $\Gamma \cong \mathbf{O}_{2}$ and Aut $\Gamma \cong \mathrm{S}_{5}$;

(2) $\Gamma \cong \mathrm{K}_{2 r}$ with $p=2 r-1$, and Aut $\Gamma \cong \mathrm{S}_{2 r}$;

(3) $\Gamma \cong \mathrm{K}_{r, r}$ with $p=r$, and Aut $\Gamma \cong \mathrm{S}_{r}<\mathrm{S}_{2}$;

(4) $\Gamma \cong \mathrm{CD}_{2 r, p, k}$ (which, up to isomorphism, is independent of the choice of $k$ in this case), where $p \mid r-1$, and one of the following statements holds.

(i) $(r, p)=(7,3)$ and Aut $\Gamma \cong \operatorname{PGL}(2,7)$;

(ii) $(r, p)=(11,5)$ and Aut $\Gamma \cong \operatorname{PGL}(2,11)$;

(iii) $(r, p) \neq(7,3)$ and $(11,5)$, and Aut $\Gamma \cong \mathrm{D}_{2 r}: \mathbb{Z}_{p}$.

Lemma 2.9 ([19, Theorem 1.1]). Let $\Gamma$ be a connected 7-valent symmetric graph of order $2 p q$ with $p>q$ odd primes. Then one of the following holds:

(1) Aut $\Gamma \cong \operatorname{PSL}(2, p)$ with $p \geq 13$;

(2) $q=7$ or $7|p-1,7| q-1$, and $\Gamma \cong \mathrm{CD}_{2 p q, 7, k}$ (as in Example 1.1).

\section{A lemma and an example}

In this section, we give a technical lemma and introduce an example appearing in Theorem 1.3.

The following is an assertion regarding simple groups, its proof depends on the classification of simple groups, see [12, P. 134-136].

Lemma 3.1. Let $m$ be an odd square-free integer with at least three prime factors, and let $T$ be a nonabelian simple group such that $28 \mathrm{~m}|| T \mid$ and $|T| \mid 2^{25} \cdot 3^{4} \cdot 5^{2} \cdot 7 \mathrm{~m}$. Then the couple $(T,|T|)$ is listed in Table 4 , where $p$ in part 4 is the largest prime factor of $m$ and $p \geq 13$.

Proof. If $T$ is a sporadic simple group, by [12, P. 135-136], $T=\mathrm{M}_{22}, \mathrm{M}_{23}, \mathrm{M}_{24}, \mathrm{~J}_{1}$, HS or Ru, as in part 1 of Table 4 . If $T=\mathrm{A}_{n}$ is an alternating group, since $3^{6}$ does not divide $|T|$ and $3^{6}|| \mathrm{A}_{15} \mid$, we have $n \leq 14$, it then easily follows that $T=\mathrm{A}_{11}, \mathrm{~A}_{12}, \mathrm{~A}_{13}$ or $\mathrm{A}_{14}$, as in part 2 of Table 4.

Now, suppose that $T$ is a simple group of Lie type defined on the $r^{e}$-elements field $\mathrm{GF}\left(r^{e}\right)$, where $r$ is a prime. If $T$ is of exceptional Lie type, by [12, P. 135], $T \cong \mathrm{Sz}(512)$ or ${ }^{3} \mathrm{D}_{4}(2)$, as listed in part 3 of Table 4 . Consider the case where $T$ is of classical Lie type. Since $r^{e}|| T \mid$, we have that $e=1$ if $r>7$, and $e \leq 2$ if $r=7$, by [12, P. 135], which give rise to examples $T=\operatorname{PSL}(2, p)$ with $p \geq 13$ a prime (noting that $\operatorname{PSL}(2, p)$ with $p=5,7,11$ does not satisfy the hypothesis of Lemma 3.1) and $T=\operatorname{PSL}(2,49)$. If $r=5$, as $5^{4} \backslash|T|$, we conclude from [12, P. 135] that $T=\operatorname{PSL}(2,125)$. For the case where $r \leq 3$, since $3^{6}, 5^{4}$ and $7^{3}$ do not divide $|T|$, by [12, P. 135] and with the help of Magma [1], we conclude that $T$ is isomorphic to one of the groups listed in part 4 of Table 4 .

Given a permutation group $G$, a direct computation by Magma program [1] can determine all orbital graphs of $G$ (see [7, P. 66] for the definition of orbital graph), or in other words, can determine all symmetric graphs which admit $G$ as an arc-transitive automorphism group. It is then easy to have the following example.

Example 3.2. There is a unique connected 7 -valent symmetric graph of order 330 , denoted by $\mathcal{C}_{330}$, which admits $\mathrm{M}_{22}$ or $\mathrm{M}_{22} \cdot \mathbb{Z}_{2}$ as an arc-transitive automorphism group. The graph $\mathcal{C}_{330}$ satisfies the conditions in Row 2 of Table 1 . 
Table 4: Nonabelian simple groups $T$ with $28 m|| T \mid$ and $|T| \mid 2^{25} \cdot 3^{4} \cdot 5^{2} \cdot 7 m$.

\begin{tabular}{|c|c|c|}
\hline Part & $T$ & $|T|$ \\
\hline \multirow[t]{6}{*}{1} & $\mathrm{M}_{22}$ & $2^{7} \cdot 3^{2} \cdot 5 \cdot 7 \cdot 11$ \\
\hline & $\mathrm{M}_{23}$ & $2^{7} \cdot 3^{2} \cdot 5 \cdot 7 \cdot 11 \cdot 23$ \\
\hline & $\mathrm{M}_{24}$ & $2^{10} \cdot 3^{3} \cdot 5 \cdot 7 \cdot 11 \cdot 23$ \\
\hline & $\mathrm{J}_{1}$ & $2^{3} \cdot 3 \cdot 5 \cdot 7 \cdot 11 \cdot 19$ \\
\hline & HS & $2^{9} \cdot 3^{2} \cdot 5^{3} \cdot 7 \cdot 11$ \\
\hline & $\mathrm{Ru}$ & $2^{14} \cdot 3^{3} \cdot 5^{3} \cdot 7 \cdot 13 \cdot 29$ \\
\hline \multirow[t]{4}{*}{2} & $\mathrm{~A}_{11}$ & $2^{7} \cdot 3^{4} \cdot 5^{2} \cdot 7 \cdot 11$ \\
\hline & $\mathrm{A}_{12}$ & $2^{9} \cdot 3^{5} \cdot 5^{2} \cdot 7 \cdot 11$ \\
\hline & $\mathrm{A}_{13}$ & $2^{9} \cdot 3^{5} \cdot 5^{2} \cdot 7 \cdot 11 \cdot 13$ \\
\hline & $\mathrm{A}_{14}$ & $2^{10} \cdot 3^{5} \cdot 5^{2} \cdot 7^{2} \cdot 11 \cdot 13$ \\
\hline \multirow[t]{2}{*}{3} & $\mathrm{Sz}(512)$ & $2^{18} \cdot 5 \cdot 7 \cdot 13 \cdot 37 \cdot 73 \cdot 109$ \\
\hline & ${ }^{3} \mathrm{D}_{4}(2)$ & $2^{12} \cdot 3^{4} \cdot 7^{2} \cdot 13$ \\
\hline \multirow[t]{26}{*}{4} & $\operatorname{PSL}(2, p)$ & $p\left(p^{2}-1\right) / 2$ \\
\hline & $\operatorname{PSL}(2,49)$ & $2^{4} \cdot 3 \cdot 5^{2} \cdot 7^{2}$ \\
\hline & $\operatorname{PSL}(2,125)$ & $2^{2} \cdot 3^{2} \cdot 5^{3} \cdot 7 \cdot 31$ \\
\hline & $\operatorname{PSL}\left(2,2^{6}\right)$ & $2^{6} \cdot 3^{2} \cdot 5 \cdot 7 \cdot 13$ \\
\hline & $\operatorname{PSL}\left(2,2^{9}\right)$ & $2^{9} \cdot 3^{3} \cdot 7 \cdot 19 \cdot 73$ \\
\hline & $\operatorname{PSL}\left(2,2^{12}\right)$ & $2^{12} \cdot 3^{2} \cdot 5 \cdot 7 \cdot 13 \cdot 17 \cdot 241$ \\
\hline & $\operatorname{PSL}\left(2,2^{15}\right)$ & $2^{15} \cdot 3^{2} \cdot 7 \cdot 11 \cdot 31 \cdot 151 \cdot 331$ \\
\hline & $\operatorname{PSL}\left(2,2^{18}\right)$ & $2^{18} \cdot 3^{3} \cdot 5 \cdot 7 \cdot 13 \cdot 19 \cdot 37 \cdot 73 \cdot 109$ \\
\hline & $\operatorname{PSL}\left(2,2^{21}\right)$ & $2^{21} \cdot 3^{2} \cdot 7^{2} \cdot 43 \cdot 127 \cdot 337 \cdot 5419$ \\
\hline & $\operatorname{PSL}\left(2,2^{24}\right)$ & $2^{24} \cdot 3^{2} \cdot 5 \cdot 7 \cdot 13 \cdot 17 \cdot 97 \cdot 241 \cdot 257 \cdot 673$ \\
\hline & $\operatorname{PSL}(3,8)$ & $2^{9} \cdot 3^{2} \cdot 7^{2} \cdot 73$ \\
\hline & $\operatorname{PSL}(3,16)$ & $2^{12} \cdot 3^{2} \cdot 5^{2} \cdot 7 \cdot 13 \cdot 17$ \\
\hline & $\operatorname{PSL}(3,64)$ & $2^{18} \cdot 3^{4} \cdot 5 \cdot 7^{2} \cdot 13 \cdot 19 \cdot 73$ \\
\hline & $\operatorname{PSL}(4,4)$ & $2^{12} \cdot 3^{4} \cdot 5^{2} \cdot 7 \cdot 17$ \\
\hline & $\operatorname{PSL}(5,2)$ & $2^{10} \cdot 3^{2} \cdot 5 \cdot 7 \cdot 31$ \\
\hline & $\operatorname{PSL}(5,4)$ & $2^{20} \cdot 3^{5} \cdot 5^{2} \cdot 7 \cdot 11 \cdot 17 \cdot 31$ \\
\hline & $\operatorname{PSL}(6,2)$ & $2^{15} \cdot 3^{4} \cdot 5 \cdot 7^{2} \cdot 31$ \\
\hline & $\operatorname{PSL}(7,2)$ & $2^{21} \cdot 3^{4} \cdot 5 \cdot 7^{2} \cdot 31 \cdot 127$ \\
\hline & $\operatorname{PSp}(6,4)$ & $2^{18} \cdot 3^{4} \cdot 5^{3} \cdot 7 \cdot 13 \cdot 17$ \\
\hline & $\operatorname{PSp}(8,2)$ & $2^{16} \cdot 3^{5} \cdot 5^{2} \cdot 7 \cdot 17$ \\
\hline & $\operatorname{PSp}(4,8)$ & $2^{12} \cdot 3^{4} \cdot 5 \cdot 7^{2} \cdot 13$ \\
\hline & $\mathrm{P} \Omega(7,4)$ & $2^{18} \cdot 3^{4} \cdot 5^{3} \cdot 7 \cdot 13 \cdot 17$ \\
\hline & $\mathrm{P} \Omega(9,2)$ & $2^{16} \cdot 3^{5} \cdot 5^{2} \cdot 7 \cdot 17$ \\
\hline & $\mathrm{P} \Omega^{+}(10,2)$ & $2^{20} \cdot 3^{5} \cdot 5^{2} \cdot 7 \cdot 17 \cdot 31$ \\
\hline & $\mathrm{P} \Omega^{-}(8,2)$ & $2^{12} \cdot 3^{4} \cdot 5 \cdot 7 \cdot 17$ \\
\hline & $\mathrm{P} \Omega^{-}(8,4)$ & $2^{24} \cdot 3^{4} \cdot 5^{3} \cdot 7 \cdot 13 \cdot 17 \cdot 257$ \\
\hline
\end{tabular}




\section{Proof of Theorem 1.2}

In this section, we prove Theorem 1.2 which in particular gives a partial proof of Theorem 1.3.

Let $\Gamma$ be a connected symmetric graph of odd prime valency $p$ and square-free order $n$, and let $G$ be a soluble arc-transitive automorphism group of $\Gamma$. Since $\Gamma$ is of odd valency, $n$ is even. Set $n=2 p_{1} p_{2} \cdots p_{t}$, with $p_{1}, p_{2}, \ldots, p_{t}$ distinct odd primes.

If $t=1$, by Lemma $2.8, \Gamma \cong \mathrm{CD}_{2 p_{1}, p, k}$ (as in part (2) of Theorem 1.2) or $\Gamma \cong \mathrm{K}_{p, p}$. If $\Gamma \cong \mathrm{K}_{p, p}$, then $G \leq$ Aut $\Gamma \cong \mathrm{S}_{p} \prec \mathbb{Z}_{2}$, as $G$ is arc-transitive on $\Gamma, \mathbb{Z}_{p}^{2} \triangleleft G$ and $G \nsubseteq \mathrm{S}_{p}^{2}$, it is then routine to show that $G \cong\left(\left(\left(\mathbb{Z}_{p}: \mathbb{Z}_{l_{1}}\right) \times\left(\mathbb{Z}_{p}: \mathbb{Z}_{l_{2}}\right)\right) \cdot \mathbb{Z}_{r}\right) \cdot \mathbb{Z}_{2}$, where $l_{i} r \mid p-1$ for $i=1,2$, as in part (1) of Theorem 1.2.

Suppose $t \geq 2$ in the following. Let $F$ be the Fitting subgroup of $G$. By Lemma 2.2, $F \neq 1$. As $|V \Gamma|=2 p_{1} p_{2} \cdots p_{t}, G$ has no nontrivial normal Sylow $a$-subgroup, where $a \notin\left\{2, p_{1}, p_{2}, \ldots, p_{t}\right\}$ is a prime, hence

$$
F=\mathbf{O}_{2}(G) \times \mathbf{O}_{p_{1}}(G) \times \cdots \times \mathbf{O}_{p_{t}}(G),
$$

where $\mathbf{O}_{2}(G)$ and $\mathbf{O}_{p_{i}}(G)$ with $i=1,2, \ldots, t$ denote the largest normal 2- and $p_{i}$ subgroups of $G$, respectively.

For each prime $q \in\left\{2, p_{1}, p_{2}, \ldots, p_{t}\right\}$, since $t \geq 2, \mathbf{O}_{q}(G)$ has at least six orbits on $V \Gamma$, by Theorem 2.7, $\mathbf{O}_{q}(G)$ is semi-regular on $V \Gamma$, so is $F$ and $\mathbf{O}_{q}(G) \leq \mathbb{Z}_{q}$. Hence $F \leq \mathbb{Z}_{n}$ is cyclic and $C_{G}(F)=F$ by Lemma 2.2.

If $F$ is transitive on $V \Gamma$, then $F \cong \mathbb{Z}_{n}$ is regular on $V \Gamma$ and $\Gamma$ is a Cayley graph of $F$. Set $\Gamma=\operatorname{Cay}(F, S)$, where $S=S^{-1} \subseteq F \backslash\{1\}$ with size $|S|=p$. Since $F \triangleleft G$, by [11, Lemma 2.9], $G \leq F$ :Aut $(F, S)$, so $\mathrm{G}_{\mathbf{1}} \leq \operatorname{Aut}(F, S) \leq \operatorname{Aut}(F)$ is transitive on $\Gamma(\mathbf{1})=S$, where 1 denotes the vertex of $\Gamma$ corresponding to the identity element of $F$, thus elements in $S$ have the same order, say $h$. Clearly, $h \neq 2$ as $F$ has a unique involution. If $h>2$, as $S=S^{-1},|S|$ is even, which is a contradiction.

If $F$ has at least three orbits on $V \Gamma$, then Theorem 2.7 implies that the normal quotient graph $\Gamma_{F}$ is $G / F$-arc-transitive; however, by Lemma 2.2, $G / F=G / C_{G}(F) \leq \operatorname{Aut}(F)$ is abelian, it forces that $G / F$ is regular on $V \Gamma_{F}$, and so $G / F$ is not transitive on $A \Gamma$, also yielding a contradiction.

Thus, $F$ has exactly two orbits on $V \Gamma$, and $F \cong \mathbb{Z}_{\frac{n}{2}}$. Because $t \geq 2, F$ has a nontrivial normal subgroup $K \cong \mathbb{Z}_{p_{2} p_{3} \ldots p_{t}}$. Since $K \triangleleft G$ has $2 p_{1}$ orbits on $V \Gamma$, by Theorem 2.7, $\Gamma_{K}$ is a $G / K$-arc-transitive graph of valency $p$ and order $2 p_{1}$, and $\Gamma$ is a regular $K$-cover of $\Gamma_{K}$. Such covers have been classified by [21, Theorem 1.1], hence the triple $\left(\Gamma, K, \Gamma_{K}\right)$ (as $\left(\Gamma, \mathbb{Z}_{n}, \Sigma\right)$ there) satisfies parts (1)-(5) of [21, Theorem 1.1]. Since $|K| \neq 2$, parts (1)-(3) are impossible. For part (4), since $n$ is square-free, $p_{1} \backslash|K|$, by [22, Theorem 1.1], $\Gamma \cong \mathrm{CD}_{n, p, k}$. For part (5), noting that $p_{1} \backslash|K|$, part (5)(ii) is not possible, we also have $\Gamma \cong \mathrm{CD}_{n, p, k}$. Finally, the last statement in part (2) of Theorem 1.2 is true by [9, Theorem 3.1]. This completes the proof of Theorem 1.2.

\section{Proof of Theorem 1.3}

We will prove Theorem 1.3 in this final section.

Let $\Gamma$ be a connected 7 -valent symmetric graph of square-free order $n$. Since $\Gamma$ is of odd valency, $n$ is even, so we may write

$$
n:=2 m=2 p_{1} p_{2} \ldots p_{t}
$$


where $p_{1}, p_{2}, \ldots, p_{t}$ are distinct odd primes. Let $\mathrm{A}=$ Aut $\Gamma$.

Lemma 5.1. If $t \leq 2$, then Theorem 1.3 is true.

Proof. If $t=1$, by Lemma $2.8, \Gamma \cong \mathrm{CD}_{2 p_{1}, 7, k}$ (as in part (1) of Theorem 1.3), or $\Gamma \cong$ $\mathrm{K}_{7,7}$ (as in Row 1 of Table 1 ).

If $t=2$, by Lemma 2.9, $\Gamma \cong \mathrm{CD}_{2 p_{1} p_{2}, 7, k}$ (as in part (1) of Theorem 1.3), or $\mathrm{A} \cong$ $\operatorname{PSL}(2, p)$ or $\operatorname{PGL}(2, p)$ with $p \geq 13$ a prime, satisfying part (3) of Theorem 1.3.

Thus, assume $t \geq 3$ in the following, and assume inductively that Theorem 1.3 is true for the graph which satisfies assumption of Theorem 1.3 and is of order less than $n$. Let $\alpha \in V \Gamma$. By Lemma 2.6, $\left|\mathrm{A}_{\alpha}\right| \mid 2^{24} \cdot 3^{4} \cdot 5^{2} \cdot 7$, hence $|\mathrm{A}|=\left|\mathrm{A}_{\alpha}\right||V \Gamma|$ divides $2^{25} \cdot 3^{4} \cdot 5^{2} \cdot 7 \mathrm{~m}$. Let $R$ be the soluble radical of $\mathrm{A}$, that is, the largest soluble normal subgroup of $\mathrm{A}$. Obviously, the soluble radical of $\mathrm{A} / R$ equals 1 .

The next lemma treats the case $R=1$.

Lemma 5.2. Suppose $R=1$ and $t \geq 3$. Then either Aut $\Gamma \cong \operatorname{PSL}(2, p)$ or $\operatorname{PGL}(2, p)$ with $p \geq 13$ a prime such that $p\left(p^{2}-1\right) \mid 2^{25} \cdot 3^{4} \cdot 5^{2} \cdot 7 n$, as in part (2) of Theorem 1.3 ; or $\Gamma \cong \mathcal{C}_{330}$ and Aut $\Gamma \cong \mathrm{M}_{22} .2$, as in Row 2 of Table 1 .

Proof. Let $N$ be a minimal normal subgroup of $\mathrm{A}$, and let $C=C_{\mathrm{A}}(N)$. Since $R=1$, $N=T^{d}$ and $|N|=|T|^{d}$ divides $2^{25} \cdot 3^{4} \cdot 5^{2} \cdot 7 m$, where $T$ is a nonabelian simple group and $d \geq 1$.

Claim 1. $C=1$.

Assume, on the contrary, $C \neq 1$. Then $C$ is insoluble as $R=1$. If $C$ is semi-regular on $V \Gamma$, then $|C| \mid n$, so $C$ is of square-free order and hence soluble, which is a contradiction. Thus $C_{\alpha} \neq 1$. Since $\Gamma$ is connected and $C \triangleleft \mathrm{A}$, we have $1 \neq C_{\alpha}^{\Gamma(\alpha)} \triangleleft \mathrm{A}_{\alpha}^{\Gamma(\alpha)}$, so 7||$C_{\alpha} \mid$. Arguing similarly, one may have 7||$N_{\alpha} \mid$. Now, since $N \cap C=1,\langle N, C\rangle=N \times C \triangleleft \mathrm{A}$, so $N_{\alpha} \times C_{\alpha} \triangleleft \mathrm{A}_{\alpha}$, hence $7^{2}|| \mathrm{A}_{\alpha} \mid$, which is a contradiction by Lemma 2.6. Therefore, $C=1$.

Claim 2. A is almost simple and the tuple $(T,|T|)$ is listed in Table 4.

As discussed above, 7||$N_{\alpha} \mid$. Then by Theorem 2.7, $N$ has at most two orbits on $V \Gamma$, hence $m$ divides $\left|N: N_{\alpha}\right|$, we further conclude that $7 m|| N|, 7||T|$ and $m|| T \mid$.

Without a loss of generality, let $p_{t}$ be the largest prime dividing $n$. As $t \geq 3, p_{t} \geq 7$, and as $m^{d}=\left(p_{1} p_{2} \cdots p_{t}\right)^{d}$ divides $2^{25} \cdot 3^{4} \cdot 5^{2} \cdot 7 p_{1} p_{2} \cdots p_{t}$, we have $d \leq 2$. If $d=2$, the only possibility is $t=3$ and $m=3 \cdot 5 \cdot 7$, so $|T|^{2} \mid 2^{25} \cdot 3^{5} \cdot 5^{3} \cdot 7^{2}$, hence $|T| \mid 2^{12} \cdot 3^{2} \cdot 5 \cdot 7$; recall that $m|| T \mid$, by [15, Theorem III], $T \cong \mathrm{A}_{l}$ with $l=7$ or 8 , and $N \cong \mathrm{A}_{l}^{2}$. By Claim $1, C=1$, then Lemma 2.1 implies $\mathrm{A}=\mathrm{A} / C \lesssim \operatorname{Aut}(N) \cong \mathrm{S}_{l}<\mathbb{Z}_{2}$, and as $N \cong \mathrm{A}_{l}^{2}$ is a minimal normal subgroup of $A$, we conclude that $A \cong A_{l}<\mathbb{Z}_{2},\left(A_{l}<\mathbb{Z}_{2}\right) \cdot \mathbb{Z}_{2}$ or $S_{l} \prec \mathbb{Z}_{2}$. Since $\left|\mathrm{A}_{\alpha}\right|=\frac{|\mathrm{A}|}{210}$, a direct computation by Magma [1] shows that no graph $\Gamma$ exists in this case, a contradiction. Thus, $d=1$ and $N=T$, and by Lemma 2.1, $\mathrm{A} \leq \operatorname{Aut}(T)$ is almost simple. Recall that $|T|$ divides $2^{25} \cdot 3^{4} \cdot 5^{2} \cdot 7 m$ and $7 m$ divides $|T|$, and noting that 4||$T \mid$ as $T$ is nonabelian simple, we have $28 m|| T \mid$. By Lemma 3.1, the couple $(T,|T|)$ is listed in Table 4.

Now, we will analyse all the candidates of $T$ in Table 4, thus proving Lemma 5.2. Recall that $n=2 m$ and $\left|T: T_{\alpha}\right|=m$ or $2 m$. Denote by Out $(T)$ the outer automorphism group of $T$. 
Assume $T \cong \operatorname{PSL}(2, p)$ with $p \geq 13$ a prime. Then $p\left(p^{2}-1\right) \mid 2^{25} \cdot 3^{4} \cdot 5^{2} \cdot 7 n$, and as $\operatorname{Out}(T) \cong \mathbb{Z}_{2}$ (see [12, P. 135]), we have $\mathrm{A} \cong \operatorname{PSL}(2, p)$ or $\operatorname{PGL}(2, p)$, the lemma is true.

Assume $T \cong \mathrm{M}_{22}$. Then $m=3 \cdot 5 \cdot 11=165$ and $n=330$. Since Out $\left(\mathrm{M}_{22}\right) \cong \mathbb{Z}_{2}$, $\mathrm{A} \cong \mathrm{M}_{22}$ or $\mathrm{M}_{22} \cdot \mathbb{Z}_{2}$. By Example $3.2, \Gamma \cong \mathcal{C}_{330}$, satisfying the conditions in Row 2 of Table 1.

Assume $T \cong \mathrm{M}_{23}$. Then $m=3 \cdot 11 \cdot 23,5 \cdot 11 \cdot 23$ or $3 \cdot 5 \cdot 11 \cdot 23$. Since Out $\left(\mathrm{M}_{23}\right)=1$, $\mathrm{A}=T \cong \mathrm{M}_{23}$ and so $\left|T: T_{\alpha}\right|=2 m=1518,2530$ or 7590 . However, by [4], $\mathrm{M}_{23}$ has no subgroup with index 1518,2530 or 7590 , a contradiction.

Assume $T \cong \mathrm{J}_{1}$. Then $m=627,1045$ or 3135 . Since Out $\left(\mathrm{J}_{1}\right)=1$, we have $\mathrm{A}=T$ and $\left|T: T_{\alpha}\right|=2 m=1254,2090$ or 6270 . By [4], $J_{1}$ has no subgroup with index 1254,2090 or 6270 , which is a contradiction.

Suppose $T \cong \mathrm{A}_{12}$. Then $m=165$ and $\left|T: T_{\alpha}\right|=165$ or 330. By [4], $\mathrm{A}_{12}$ has no subgroup with index 165 or 330 , yielding a contradiction.

Suppose $T \cong \operatorname{PSL}(2,49)$. Then $m=105$ and $\left|T: T_{\alpha}\right|=105$ or 210 , it follows $\left|T_{\alpha}\right|=560$ or 280 respectively. By Lemma $2.3, \operatorname{PSL}(2,49)$ has no subgroup with order 560 or 280 , a contradiction.

Suppose $T \cong \operatorname{PSL}\left(2,2^{24}\right)$. Then Out $(T) \cong \mathbb{Z}_{24}$ by [12, P. 135], it follows $A \cong$ $\operatorname{PSL}\left(2,2^{24}\right) \cdot \mathbb{Z}_{r}$ with $r \mid 24$, and $|\mathrm{A}|=2^{24} \cdot 3^{2} \cdot 5 \cdot 7 \cdot 13 \cdot 17 \cdot 97 \cdot 241 \cdot 257 \cdot 673 r$. Hence $m=$ $3 \cdot 13 \cdot 17 \cdot 97 \cdot 241 \cdot 257 \cdot 673,5 \cdot 13 \cdot 17 \cdot 97 \cdot 241 \cdot 257 \cdot 673$ or $3 \cdot 5 \cdot 13 \cdot 17 \cdot 97 \cdot 241 \cdot 257 \cdot 673$. For the first case, $\left|\mathrm{A}_{\alpha}\right|=2^{23} \cdot 3 \cdot 5 \cdot 7 \mathrm{r}$, which is impossible by Lemma 2.6. For the second case, $\left|\mathrm{A}_{\alpha}\right|=2^{23} \cdot 3^{2} \cdot 7 r$, by Lemma 2.6 , the only possibility is $r=2$ and $\mathrm{A}_{\alpha} \cong \mathbb{Z}_{2}^{20}:(\operatorname{SL}(2,2) \times$ $\mathrm{SL}(3,2))$; for the last case, we have $\left|\mathrm{A}_{\alpha}\right|=2^{23} \cdot 3 \cdot 7 r$, by Lemma 2.6 , the only possibility is $r=6$ and $\mathrm{A}_{\alpha} \cong \mathbb{Z}_{2}^{20}:(\mathrm{SL}(2,2) \times \mathrm{SL}(3,2))$. However, by Lemma 2.3 , both $\operatorname{PSL}\left(2,2^{24}\right) \cdot \mathbb{Z}_{2}$ and $\operatorname{PSL}\left(2,2^{24}\right) \cdot \mathbb{Z}_{6}$ have no subgroup isomorphic to $\mathbb{Z}_{2}^{20}:(\operatorname{SL}(2,2) \times \operatorname{SL}(3,2))$, which is a contradiction.

Suppose $T \cong{ }^{3} \mathrm{D}_{4}(2)$. Since Out $(T) \cong \mathbb{Z}_{3}$ (see [4]), $\mathrm{A} \cong{ }^{3} \mathrm{D}_{4}(2)$ or ${ }^{3} \mathrm{D}_{4}(2) . \mathbb{Z}_{3}$, and so $|\mathrm{A}|=2^{12} \cdot 3^{4} \cdot 7^{2} \cdot 13$ or $2^{12} \cdot 3^{5} \cdot 7^{2} \cdot 13$ respectively, implying $m=3 \cdot 7 \cdot 13$. Now, $\left|\mathrm{A}_{\alpha}\right|=\frac{|\mathrm{A}|}{2 m}=2^{11} \cdot 3^{3} \cdot 7$ or $2^{11} \cdot 3^{4} \cdot 7$, which is impossible by Lemma 2.6 .

Arguing similarly as above, one may prove that no graph $\Gamma$ exists for all other candidates for $T$ in Table 4 (the results have been checked by Magma [1]).

We finally consider the case where $\mathrm{A}$ is insoluble and $R \neq 1$ by the following lemma.

Lemma 5.3. Suppose that $\mathrm{A}$ is insoluble, $R \neq 1$ and $t \geq 3$. Then no graph $\Gamma$ exists.

Proof. Let $M$ be a minimal soluble normal subgroup of A. Then $M \cong \mathbb{Z}_{r}^{d}$, where $r$ is a prime and $d \geq 1$. Since $t \geq 3, M$ has at least $2 \cdot 3 \cdot 5=30$ orbits on $V \Gamma$, so, by Theorem 2.7, $M$ is semi-regular on $V \Gamma$ (so $d=1$ and $r \in\left\{2, p_{1}, p_{2}, \ldots, p_{t}\right\}$ ), $\Gamma_{M}$ is a 7 -valent A/M-arc-transitive graph of order $\frac{2 m}{r}$, and $\Gamma$ is an arc-transitive regular $\mathbb{Z}_{r}$-cover of $\Gamma_{M}$.

If $r=2$, then $\Gamma_{M}$ is arc-transitive of odd order $m$ and odd valency 7, which is impossible.

Thus, $r=p_{i}$ with $i \in\{1,2, \ldots, t\}$, and $\Gamma_{M}$ is a 7 -valent $\mathrm{A} / M$-arc-transitive graph of order $\frac{2 m}{p_{i}}$. Recall that we assume by inductive hypothesis that Theorem 1.3 is true for all graphs which satisfy the assumptions of Theorem 1.3 and are of order less than $n$, so $\Gamma_{M}$ satisfies Theorem 1.3. Noting that $\mathrm{A}$ is insoluble and $M$ is soluble, $\mathrm{A} / M$ is insoluble, so is Aut $\left(\Gamma_{M}\right)$. Then, checking the graphs in Theorem 1.3, we conclude that the soluble radical of Aut $\left(\Gamma_{M}\right)$ equals 1 , and one of the following holds: 
(1) $\Gamma_{M} \cong \mathcal{C}_{330}$ and $\operatorname{Aut}\left(\Gamma_{M}\right) \cong \mathrm{M}_{22} \cdot \mathbb{Z}_{2}$;

(2) $\operatorname{Aut}\left(\Gamma_{M}\right) \cong \operatorname{PSL}(2, p)$ or $\operatorname{PGL}(2, p)$ with $p \geq 13$ a prime and $t \geq 3$.

Since $\mathrm{A} / M$ acts arc-transitively on $\Gamma_{M}$, we have $7\left|V \Gamma_{M}\right|$ divides $|\mathrm{A} / M|$.

For case (1), then $\left|V \Gamma_{M}\right|=330$, and $7 \cdot 330=2310$ divides $|\mathrm{A} / M|$, since $\mathrm{A} / M \leq$ $\mathrm{M}_{22} \cdot \mathbb{Z}_{2}$, by [4], we have $\mathrm{A} / M \cong \mathrm{M}_{22}$ or $\mathrm{M}_{22} \cdot \mathbb{Z}_{2}$. Let $X$ be a normal subgroup of $\mathrm{A}$ such that $X \cong M \cdot \mathrm{M}_{22} \cong \mathbb{Z}_{r} \cdot \mathrm{M}_{22}$. Since $\left(r,\left|V \Gamma_{M}\right|\right)=(r, 330)=1$, we have $r \neq 2$ and 3 . Then, as $\mid$ Mult $\left(\mathrm{M}_{22}\right) \mid=12$ (see [4]), Lemma 2.5 implies $X \cong \mathbb{Z}_{r} \times \mathrm{M}_{22}$ and $X^{\prime} \cong \mathrm{M}_{22}$. Since $\left|X^{\prime}\right|=\left|\mathrm{M}_{22}\right|$ does not divide $|V \Gamma|, X^{\prime} \triangleleft \mathrm{A}$ is not semi-regular on $V \Gamma$, and $X^{\prime}$ has at most two orbits on $V \Gamma$ by Theorem 2.7. Because $\Gamma$ is connected and $1 \neq X_{\alpha}^{\prime} \triangleleft \mathrm{A}_{\alpha}$, $1 \neq\left(X_{\alpha}^{\prime}\right)^{\Gamma(\alpha)} \triangleleft \mathrm{A}_{\alpha}^{\Gamma(\alpha)}$, it follows 7||$X_{\alpha}^{\prime} \mid$. Hence $r$ divides $\frac{\left|X^{\prime}\right|}{7}=2^{7} \cdot 3^{2} \cdot 5 \cdot 11$, which is a contradiction as $\left(r,\left|V \Gamma_{M}\right|\right)=(r, 330)=1$.

We next consider case (2). Since $\mathrm{A} / M$ is insoluble and 7|| $\mathrm{A} / M \mid$, by Lemma 2.4, $\mathrm{A} / M \cong \mathrm{PSL}(2, p)$ or $\mathrm{PGL}(2, p)$. Let $B / M \triangleleft \mathrm{A} / M$ such that $B / M \cong \mathrm{PSL}(2, p)$. Since Mult(PSL $(2, p)) \cong \mathbb{Z}_{2}$ (see [12, P. 302]) and $r \geq 3$, Lemma 2.5 implies that $B^{\prime} \cong$ $\operatorname{PSL}(2, p)$ and $B=M \times B^{\prime}$. Since $B, B^{\prime} \triangleleft A$ are insoluble, both $B$ and $B^{\prime}$ have at most two orbits on $V \Gamma$. In particular, $m$ divides $\left|B^{\prime}\right|$.

If $r>7$, since $|\mathrm{A}|$ divides $2^{25} \cdot 3^{4} \cdot 5^{2} \cdot 7 m$, and $|B|=\left|M \times B^{\prime}\right|=r\left|B^{\prime}\right|$ divides $|\mathrm{A}|$, we have $r \nmid\left|B^{\prime}\right|$, which is a contradiction to $m$ dividing $\left|B^{\prime}\right|$.

Assume $r=7$. Since $\Gamma$ is connected and $1 \neq B_{\alpha}^{\prime} \triangleleft \mathrm{A}_{\alpha}$, we have 7||$B_{\alpha}^{\prime} \mid$. Then, as $\left|B^{\prime}: B_{\alpha}^{\prime}\right|=m$ or $2 m$ is divisible by 7 , we further conclude that $7^{2}$ divides $\left|B^{\prime}\right|=$ $|B / M|$. However, since $\left|B / M:(B / M)_{\delta}\right|=\frac{m}{7}$ or $\frac{2 m}{7}$, which is not divisible by 7 , we have $7^{2}||(B / M)_{\delta} \mid$, so $7^{2}||(\mathrm{A} / M)_{\delta} \mid$, which is a contradiction by Lemma 2.6.

Assume finally $r=3$ or 5 . Since $B / M \cong B^{\prime}$ has at most two orbits on $V \Gamma_{N}$, and $B^{\prime}$ has at most two orbits on $V \Gamma$, we have $\left|B / M:(B / M)_{\delta}\right|=\frac{m}{r}$ or $\frac{2 m}{r}$, and $\left|B^{\prime}: B_{\alpha}^{\prime}\right|=m$ or $2 m$. It follows that $r||(B / M)_{\delta} \mid$ and so $r||(\mathrm{A} / M)_{\delta} \mid$. Also, as $\mathrm{A} / M$ acts arc-transitively on $\Gamma_{M}, 7||(\mathrm{A} / M)_{\delta} \mid$, hence $7 r||(\mathrm{A} / M)_{\delta} \mid$. Suppose $r=3$. If $(\mathrm{A} / M)_{\delta}$ is soluble, then $(\mathrm{A} / M)_{\delta}$ is listed in part (1) of Lemma 2.6, and as 21||$(\mathrm{A} / M)_{\delta} \mid$, we have $(\mathrm{A} / M)_{\delta} \geq \mathrm{F}_{21}$; however, since $\mathrm{A} / M \leq \operatorname{PGL}(2, p)$ and $p \neq 7$, by Lemma $2.4, \operatorname{PGL}(2, p)$ has no soluble subgroup containing a subgroup isomorphic to $\mathrm{F}_{21}$, a contradiction. If $(\mathrm{A} / M)_{\delta}$ is insoluble, noting that $\mathrm{A} / M \leq \mathrm{PGL}(2, p)$, we have $(\mathrm{A} / M)_{\delta} \cong \mathrm{PSL}(2, p)$ or $\mathrm{A}_{5}$. For the first case, $\left|V \Gamma_{M}\right|=\left|\mathrm{A} / M:(\mathrm{A} / M)_{\delta}\right|=2$, which is impossible. For the latter case, $7 \nmid\left|(\mathrm{A} / M)_{\delta}\right|$, also a contradiction. Suppose now $r=5$. Then 35||$(\mathrm{A} / M)_{\delta} \mid$, and Lemma 2.6 implies that $(\mathrm{A} / M)_{\delta}$ is insoluble, so $(\mathrm{A} / M)_{\delta} \cong \mathrm{PSL}(2, p)$ or $\mathrm{A}_{5}$ as $\mathrm{A} / M \leq \mathrm{PGL}(2, p)$. Now, the same arguments as above draw a contradiction.

Theorem 1.3 now follows directly from Theorem 1.2 and Lemmas 5.1-5.3.

\section{References}

[1] W. Bosma, J. Cannon and C. Playoust, The Magma algebra system I: The user language, J. Symbolic Comput. 24 (1997), 235-265, doi:10.1006/jsco.1996.0125.

[2] P. J. Cameron, G. R. Omidi and B. Tayfeh-Rezaie, 3-designs from $\operatorname{PGL}(2, q)$, Electron. J. Combin. 13 (2006), \#R50, http: / /www. combinatorics.org/ojs/index.php/ eljc/article/view/vi3ilr50.

[3] Y. Cheng and J. Oxley, On weakly symmetric graphs of order twice a prime, J. Comb. Theory Ser. B 42 (1987), 196-211, doi:10.1016/0095-8956(87)90040-2. 
[4] J. H. Conway, R. T. Curtis, S. P. Norton, R. A. Parker and R. A. Wilson, Atlas of Finite Groups, Oxford University Press, Eynsham, 1985, http://brauer.maths.qmul.ac. uk/Atlas/v3/.

[5] L. E. Dickson, Linear Groups with an Exposition of the Galois Field Theory, Dover Publications, New York, 1958.

[6] S.-Y. Ding, B. Ling, B.-G. Lou and J.-M. Pan, Arc-transitive pentavalent graphs of square-free order, Graphs Combin. 32 (2016), 2355-2366, doi:10.1007/s00373-016-1717-8.

[7] J. D. Dixon and B. Mortimer, Permutation Groups, volume 163 of Graduate Texts in Mathematics, Springer-Verlag, New York, 1996, doi:10.1007/978-1-4612-0731-3.

[8] Y.-Q. Feng, C. H. Li and J.-X. Zhou, Symmetric cubic graphs with solvable automorphism groups, Eur. J. Combin. 45 (2015), 1-11, doi:10.1016/j.ejc.2014.10.008.

[9] Y.-Q. Feng and Y.-T. Li, One-regular graphs of square-free order of prime valency, Eur. J. Combin. 32 (2011), 265-275, doi:10.1016/j.ejc.2010.10.002.

[10] M. Giudici, C. H. Li and C. E. Praeger, Analysing finite locally $s$-arc transitive graphs, Trans. Amer. Math. Soc. 356 (2004), 291-317, doi:10.1090/s0002-9947-03-03361-0.

[11] C. D. Godsil, On the full automorphism group of a graph, Combinatorica 1 (1981), 243-256, doi:10.1007/bf02579330.

[12] D. Gorenstein, Finite Simple Groups, University Series in Mathematics, Plenum Press, New York, 1982.

[13] S.-T. Guo, Y.-T. Li and X.-H. Hua, $(G, s)$-transitive graphs of valency 7, Algebra Colloq. 23 (2016), 493-500, doi:10.1142/s100538671600047x.

[14] B. Huppert, Endliche Gruppen I, volume 134 of Grundlehren der mathematischen Wissenschaften, Springer-Verlag, Berlin, 1967, doi:10.1007/978-3-642-64981-3.

[15] B. Huppert and W. Lempken, Simple groups of order divisible by at most four primes, Proc. of the F. Skorina Gomel State Univ. 10 (2000), 64-75, www . iem. uni-due. de / lempken/ pi4.ps.

[16] C. H. Li, Z. P. Lu and G. X. Wang, Vertex-transitive cubic graphs of square-free order, J. Graph Theory 75 (2014), 1-19, doi:10.1002/jgt.21715.

[17] C. H. Li, Z. P. Lu and G. X. Wang, The vertex-transitive and edge-transitive tetravalent graphs of square-free order, J. Algebr. Comb. 42 (2015), 25-50, doi:10.1007/s10801-014-0572-z.

[18] C. H. Li and J.-M. Pan, Finite 2-arc-transitive abelian Cayley graphs, Eur. J. Combin. 29 (2008), 148-158, doi:10.1016/j.ejc.2006.12.001.

[19] B. Ling and S. Y. Ding, On 7-valent symmetric graphs of order 2pq, preprint.

[20] A. Malnič, D. Marušič and P. Potočnik, On cubic graphs admitting an edge-transitive solvable group, J. Algebr. Comb. 20 (2004), 99-113, doi:10.1023/b:jaco.0000047284.73950.bc.

[21] J.-M. Pan, Z.-H. Huang and S.-Y. Ding, Arc-transitive cyclic covers of graphs with order twice a prime, Discrete Math. 340 (2017), 811-816, doi:10.1016/j.disc.2016.11.018.

[22] J.-M. Pan, Z.-H. Huang and Z. Liu, Arc-transitive regular cyclic covers of the complete bipartite graph $\mathrm{K}_{p, p}$, J. Algebr. Comb. 42 (2015), 619-633, 10.1007 / s10801-015-0594-1.

[23] J.-M. Pan, Y. Liu, Z.-H. Huang and C.-L. Liu, Tetravalent edge-transitive graphs of order $p^{2} q$, Sci. China Math. 57 (2014), 293-302, doi:10.1007/s11425-013-4708-8.

[24] C. E. Praeger, An O'Nan-Scott theorem for finite quasiprimitive permutation groups and an application to 2-arc transitive graphs, J. London Math. Soc. 47 (1993), 227-239, doi:10.1112/ jlms/s2-47.2.227. 
[25] J. Schur, Untersuchungen über die Darstellung der endlichen Gruppen durch gebrochene lineare Substitutionen, J. Reine Angew. Math. 132 (1907), 85-137, doi:10.1515/crll.1907.132.85.

[26] M. Suzuki, Group Theory II, volume 248 of Grundlehren der mathematischen Wissenschaften, Springer-Verlag, New York, 1986, doi:10.1007/978-3-642-86885-6.

[27] R. Weiss, The nonexistence of 8-transitive graphs, Combinatorica 1 (1981), 309-311, 10 . $1007 /$ bf 02579337. 